\title{
The perception of shape and curvedness from binocular stereopsis and structure from motion
}

\author{
JAMES S. TITTLE and VICTOR J. PEROTTI \\ Ohio State University, Columbus, Ohio
}

\begin{abstract}
The integration of binocular stereopsis and kinetic depth was measured for two distinct aspects of 3-D structure: (1) shape index, which is a measure of scale-independent structure, and (2) curvedness, which is a measure of scale-dependent structure. We found that motion contributes significantly more to judged shape index than it does to judged curvedness, and stereo contributes significantly more to judged curvedness than it does to judged shape index. This suggests that the differences in the relative contribution of motion and stereo reported here occurred because these two sources do not equally specify the scale-dependent and scale-independent aspects of surface structure. Furthermore, these results seem to be inconsistent with integration models in which the different visual cues all initially contribute to the same single representation of 3-D structure.
\end{abstract}

Our perception of an object or surface consists of many different properties, such as its distance, size, and shape. Although the first two of these attributes can be defined in a relatively straightforward manner, there is not uniform agreement about what it means to perceive the shape of an object or surface (e.g., it could be its depth, orientation, curvature, or some combination of properties). Distinguishing between these possibilities is particularly important when considering the different types of retinal information available to specify three-dimensional (3-D) shape. For example, theoretical analyses demonstrate that while relative depth can be recovered directly from binocular disparities, it is difficult, if not impossible, to recover this information directly from shading or texture. In the case of texture and shading, it is more likely that the visual system instead recovers relative slant or curvature without first computing local depth. Because of these potential differences, a full understanding of 3-D shape perception must entail not only a description of those properties represented by the visual system but also the degree to which each is specified by the various optical sources of information. The purpose of the experiments presented here was to examine the integration of different surface properties from two of the more salient sources of 3-D structure: motion and binocular stereopsis.

To provide an example of some of the candidates for local shape descriptors, consider a quadric surface patch of the form

$$
Z(x, y)=\frac{1}{2}\left(k_{1} x_{2}+k_{2} y_{2}\right)
$$

The authors would like to thank Farley Norman, Flip Philips, and James Todd for helpful comments and discussions regarding the issues described in this paper. Correspondence should be addressed to J. S. Tittle, Department of Psychology, Ohio State University, 142 Townshend Hall, Columbus, OH 43210-1222 (e-mail: jtittle@magnus.acs. ohiostate.edu). where $Z$ is depth along the line of sight, and $x$ and $y$ represent coordinates in the image plane. Traditionally, psychologists have sought to describe the shape of such a patch using a quantitative measure of each local region on the surface. The three most commonly proposed local measures have been (1) depth, (2) orientation, and (3) curvature. Figure 1 provides a schematic view of these three local property maps and organizes them on the basis of their level of differential surface structure: zeroth order, first order, and second order.

The most basic representation of the shape of this surface patch would be the set of $(x, y, z)$ values satisfying Equation 1 in a coordinate system with the origin at the eye. Such a representation, presented schematically on the top of Figure 1, is commonly referred to as a depth map. The depth map is said to represent zeroth-order surface structure because it does not take into account how the surface changes around each local measurement. Although a depth map may provide a suitable description of shape for many tasks, its primary drawback is that if the observer or the object moves, the representation will change almost everywhere even though the object remains unchanged. This lack of stability can be partially avoided by using an object-centered, rather than a viewer-centered, coordinate system; however, the fact remains that this type of representation is coordinate system dependent. Furthermore, results showing extensive anisotropies in the perception of orientation (Gillam, Chambers, \& Russo, 1988) and curvature (Norman \& Lappin, 1992; Rogers \& Graham, 1983) are not adequately explained by a depth map.

A second potential shape descriptor is based not on the depth of visible surface regions but rather on their local orientation. At any point on a smooth surface there exists a tangent plane defined by two independent tangent lines to the surface. The unit vector perpendicular to this plane is referred to as the surface normal, and it provides a local measure of surface orientation (see middle of Figure 1). Because the orientation map describes how quickly depth 


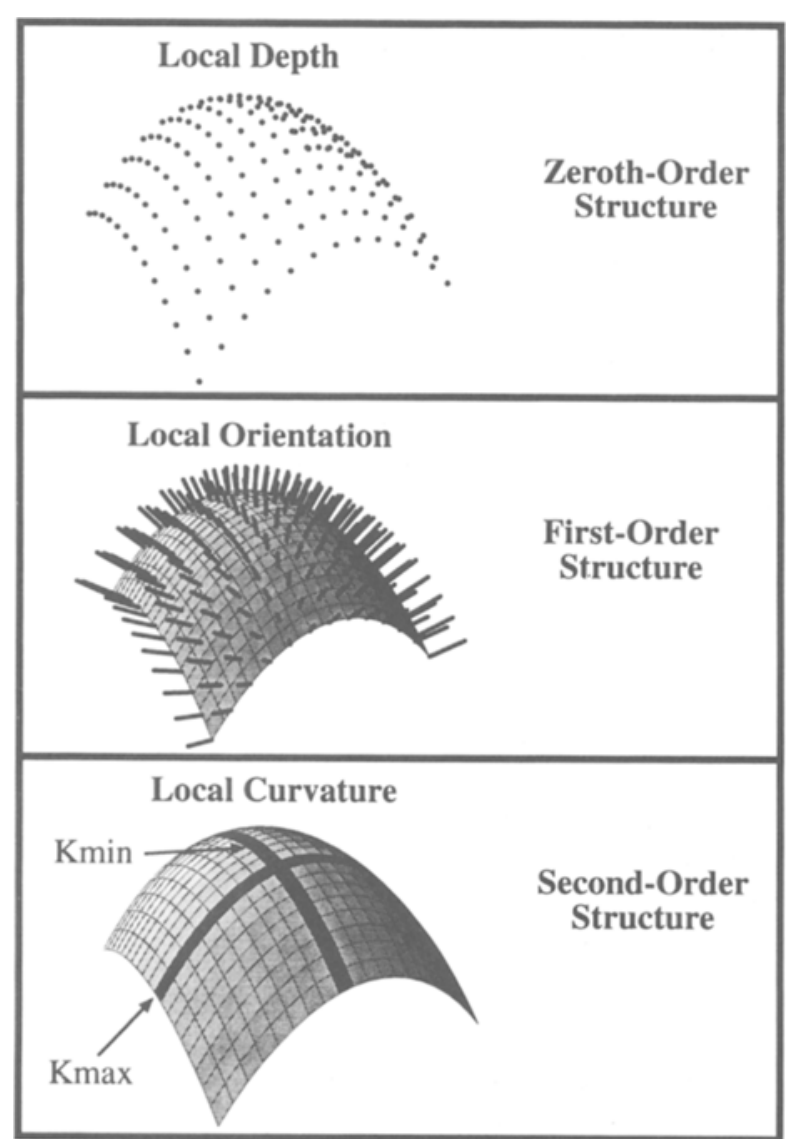

Figure 1. Three different local representations of a quadric surface patch: depth (zeroth-order), orientation (first-order), and curvature (second-order).

changes as we move along the surface (i.e., the first spatial derivative), it is considered to be a measure of firstorder surface structure. For the patch described in Equation 1 , the normal vector would be defined as follows:

$$
N(x, y)=\frac{\left(-k_{1} x,-k_{2 y}, 1\right)}{\sqrt{k_{1}^{2} x^{2}+k_{2}^{2} y^{2}+1}} .
$$

The surface normal provides information not explicitly represented in the depth map because it depends on the values of neighboring surface regions, and it is invariant under translation. Nevertheless, the surface normal is still coordinate system dependent, and, thus, it does not provide a stable representation of local shape under arbitrary rotation. This claim is supported by recent evidence suggesting that human observers are relatively insensitive to local differences in surface orientation (Todd \& Norman, 1995).

A local surface property that is not coordinate system dependent can be obtained by measuring the curvature, or the rate at which orientation changes in a particular direction along a smoothly curved surface (i.e., secondorder surface structure). For any point on a quadric (i.e., second-order) surface, there will always be one direction for which the curvature reaches a maximum $\left(k_{\max }\right)$ and an orthogonal direction for which the curvature reaches a minimum $\left(k_{\min }\right)$. These are commonly referred to as the principal curvatures, and their magnitudes are coordinate system independent. For the surface described by Equation 1, the curvatures along the $x$ and $y$ axes are given by (Kreyszig, 1959):

$$
k_{x}(x)=\frac{k_{1}}{\left(1+k_{1}^{2} x^{2}\right)^{\frac{3}{2}}}
$$

and

$$
k_{y}(y)=\frac{k_{2}}{\left(1+k_{2}^{2} y^{2}\right)^{\frac{3}{2}}} .
$$

At the origin, these are the principal curvatures, but we have replaced $k_{\max }$ and $k_{\min }$ with $k_{x}$ and $k_{y}$ because which of these two is the maximum or minimum curvature depends on the specific values of the parameters $k_{1}$ and $k_{2}$. In fact, from Equations 3 and 4, it can be seen that the principal curvatures at the origin of the quadric patch are simply $k_{1}$ and $k_{2}$ from Equation 1 (an example of these local shape descriptors is provided on the bottom of Figure 1).

Although $k_{\max }$ and $k_{\min }$ provide a complete and coordinate-system-independent description of local shape on a quadric surface, they still have certain properties that seem to be inconsistent with our perceptual experience. For example, on the basis of a $k_{\max }$ and $k_{\min }$ description of local shape, a baseball and a beach ball have representations that differ at each point on their surfaces. Nevertheless, for human observers, the fact that these two objects are both spherical would be easily detected. Thus, it should be clear that we have the ability to perceive both scale-independent (e.g., spherical vs. cylindrical) and scale-dependent (e.g., magnitude of curvature) aspects of shape, and so it would be desirable to have shape descriptors that independently represent these attributes.

Two such measures of local shape have recently been proposed by Koenderink (1990): shape index and curvedness. These measures are based on a polar coordinate transformation of $k_{\max }$ and $k_{\min }$ space to separate out their scale-independent (shape index) and scale-dependent (curvedness) components. Although these values actually vary over the entire surface, we will refer throughout the rest of the paper to the shape index and curvedness of a surface as the values defined at the origin. The shape index $(S)$ provides a 1-D description of shape independent of the magnitude of curvature:

$$
S=-\frac{2}{\pi} \arctan \frac{k_{\max }+k_{\min }}{k_{\max }-k_{\min }} .
$$

The shape index scale varies between -1 and 1 , but it is symmetric about 0 (negative values represent concave shapes and positive values represent convex ones). The top of Figure 2 shows the positive portion of the shape index scale. Because this measure is scale-independent, the cylindrical surfaces aligned vertically in Figure 2 all 


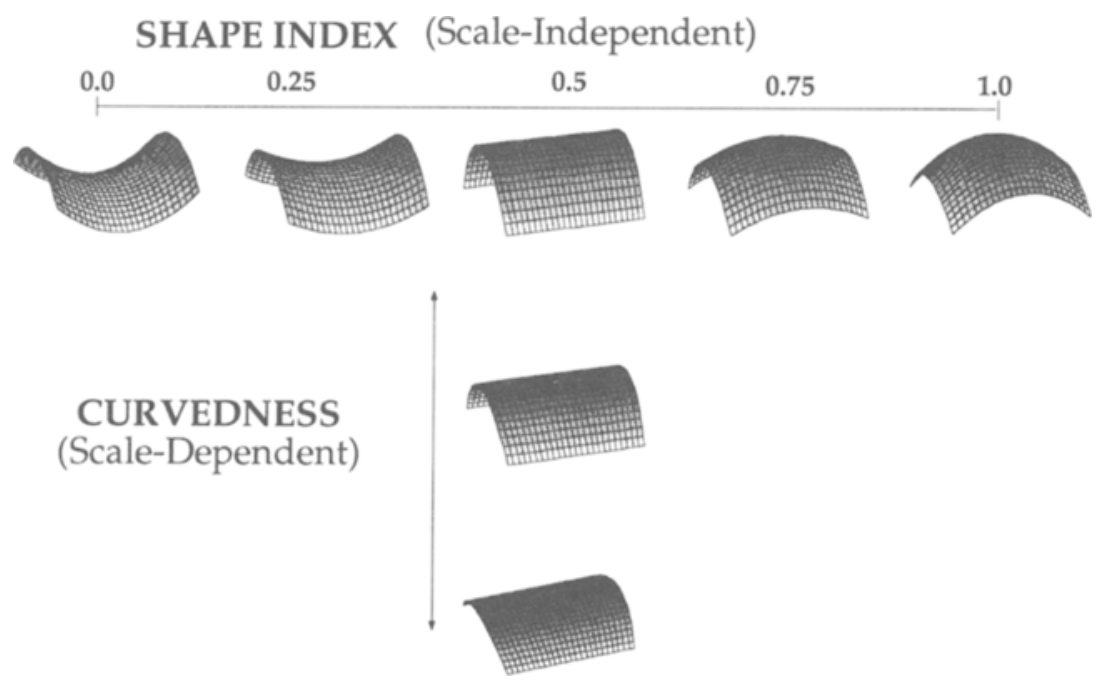

Figure 2. The shape index and curvedness scales. Shape index represents the scaleindependent aspect of 3-D structure, and curvedness represents the scale-dependent aspect.

have the same shape index value $(0.5)$. The difference in the amount of curvature between these surfaces is defined by their curvedness $(C)$ :

$$
C=\sqrt{\frac{k_{\max }^{2}+k_{\min }^{2}}{2}} .
$$

The evidence from recent studies on binocular stereopsis (de Vries, Kappers, \& Koenderink, 1993) and motion (van Damme, Oosterhoff, \& van de Grind, 1994) is consistent with the idea that human observers can accurately judge shape index independently of variations in curvedness. Such results suggest that when studying the integration of 3-D shape from multiple visual cues, one should consider both scale-dependent and scaleindependent information.

The preceding discussion does not provide an exhaustive list of local shape descriptors (for example, we have made no mention of nonmetric properties: Todd \& Reichel, 1989) but rather outlines a representative sample of possibilities. Nevertheless, these examples should serve to indicate that there are many possible properties that could be included in the representation of "shape."

When considering the integration of multiple optical sources, one important issue that follows from this discussion concerns the extent to which the various sources contribute to each of these components of surface structure. In other words, do all the sources of 3-D structure contribute equally to these measures of local shape? Some recent evidence on the perception of binocular stereopsis and kinetic depth suggests that the answer to this question is "no." Tittle, Todd, Perotti, and Norman (1995) found that perceived structure from motion exhibited failures of constancy across different orientations but not different distances, whereas perceived structure from stereo exhibited the opposite pattern of results. Such a finding is not consistent with the idea that perceived surface structure from motion and that from binocular stereopsis are based on the same local properties.
In addition, recent cross-modal matching studies between stereo and motion provide evidence that these two sources do not provide the same representations of local surface structure. Comparing cross-modal matches for surface orientation (slant) and curvature, de Vries (1993) found that perceived slant was greater for motion than for stereo but that perceived curvature was greater for stereo than for motion. Such a result is fundamentally inconsistent with the idea that perceived curvature for motion and stereo is obtained by taking the spatial derivative of local orientation, and it suggests that stereo-based curvature is computed directly. Perotti, Todd, Lappin, and Phillips (in press) also found a disassociation between structure from motion and binocular stereopsis when they performed stereo matches of motion-defined surfaces simulating different shape index and curvedness values. Specifically, they observed a very high correlation between simulated and adjusted shape index, and they observed a much lower correlation between simulated and adjusted curvedness.

This last result is particularly interesting because it suggests that structure from motion provides a much more robust measure of shape index than of curvedness. If this were the case, we would expect a difference in the relative contribution of motion and binocular stereopsis on the basis of the particular measure used to probe 3-D structure. This hypothesis was tested in the following experiments by measuring judged shape and curvedness for displays with conflicting binocular stereopsis and motion information.

\section{GENERAL METHOD}

\section{Subjects}

Four experienced psychophysical observers participated in all three experiments. These included the 2 authors (J.S.T. and V.J.P.) and 2 additional observers (J.F.N. and H.F.N.), who were unaware of the specific hypotheses under investigation. One additional observer (J.T.T.) participated only in the third experiment. All observers 
had extensive experience participating in psychophysical experiments, but only J.S.T. and V.J.P. were aware of the specific hypotheses under investigation.

\section{Apparatus and Stimuli}

The stimulus displays were presented on a Silicon Graphics Iris (4D/310 VGX) workstation. The physical resolution of the system's monitor was $1,280 \times 1,024$, but hardware antialiasing allowed us to use subpixel positioning and increase the effective resolution by a factor of 16. Left and right views of the stereoscopic stimuli were presented using LCD shuttered glasses (Stereographics) synchronized with the refresh rate of the monitor.

Our stimulus displays consisted of orthographic projections of 2,000 luminous dots randomly placed on stereoscopically viewed, rotating surfaces. All the surfaces used in our experiments satisfied Equation 1 and varied only in terms of the specific values chosen for the parameters $k_{1}$ and $k_{2}$. On any given trial, these parameter values were chosen by solving Equations 5 and 6 for a particular shape index and curvedness. Specifically, the shape index varied between 0.0 and 1.0 (as shown at the top of Figure 2), and the curvedness varied between $0.25 \mathrm{~cm}^{-1}$ and $1.25 \mathrm{~cm}^{-1}$ (see Figures 3 and 5 for example stereograms). To provide some idea of the depth range simulated in our quadric surfaces, the maximum depth difference between the high-curvedness $\left(1.25 \mathrm{~cm}^{-1}\right)$ and lowcurvedness $\left(0.25 \mathrm{~cm}^{-1}\right)$ surfaces, with shape index 1.0 , was approximately $8.0 \mathrm{~cm}$.

The dots on these surfaces were 4 square pixels in size and subtended a visual angle of $0.03^{\circ}$ at our viewing distance of $114 \mathrm{~cm}$. Kinetic depth was provided by oscillating the surfaces $\pm 15^{\circ}$ about a vertical axis passing through the origin. The frame rate for our motion sequences was $30 \mathrm{~Hz}$, and the surface rotated at a constant angular velocity that was randomly chosen for each trial from a uniform distribution varying between $0.5^{\circ}$ and $1.5^{\circ}$ per frame. To eliminate the appearance of deforming surface boundaries as the object rotated, we simulated a square aperture that was stereoscopically placed in front of the surface. Through this aperture, the visible region of the surface subtended $6.5^{\circ}$ of visual angle in both the vertical and the horizontal dimensions.

Binocular disparities were created in the displays by presenting separate views to the left and right eyes on the basis of an interocular separation of $6.25 \mathrm{~cm}$ and a viewing distance of $114 \mathrm{~cm}$. The relative binocular disparity at the origin of the surface was always zero, and (for a frontoparallel view of our surfaces) it varied up to a maximum of approximately $6^{\prime}$ of arc for the largest curvedness value used.

The first step in creating the stereo-motion conflict displays was to randomly choose $x$-and $y$-coordinates for the dots that would be placed on the surface. Creating a conflict surface then simply requires assigning two $z$-coordinates-one for the motion surface and one for the stereo- to each unique $(x, y)$ pair. We accomplished this by randomly choosing two sets of $k_{1}$ and $k_{2}$ values on each trial: One set of the parameters would then be used to define the motion surface $z$-coordinates $\left(z_{\text {motion }}\right)$ and the other would define the stereo surface $z$-coordinates $\left(z_{\text {stereo }}\right)$. This resulted in a set of dots that occupied the same positions on the monitor but defined a unique motion surface $\left(x, y, z_{\text {motion }}\right)$ and stereo surface $\left(x, y, z_{\text {stereo }}\right)$.

To simultaneously depict these two surfaces then required applying the appropriate 3-D transformation, for either motion or binocular viewing, to the appropriate set of $(x, y, z)$ coordinates. As a result of this process, we got a velocity for each dot based on ( $x$, $\left.y, z_{\text {motion }}\right)$ and a binocular disparity for each dot based on $(x, y$, $\left.z_{\text {stereo }}\right)$. This procedure works fine for the first frame, but, as the dots move, their new $(x, y)$ coordinates no longer match the correct $z_{\text {stereo }}$ for the stereo surface. Thus, after every frame, we recalculated the $z_{\text {stereo }}$ of each dot based on its new $(x, y)$ coordinates. Also, as the motion surface rotates out of the frontoparallel plane, the stereo surface must rotate by the same amount. Otherwise, we would have simulated a static stereo surface viewed along with our motion sur- face. To correct this problem, we computed $z_{\text {stereo }}$ based on a transformed version of Equation 1 that took into account the rotation of the motion surface.

The result of the procedure outlined above is that we were able to create displays in which the relative motion of dots defined one surface, and the binocular disparity of dots defined a different surface. However, one potential drawback of this technique is that it introduces small perturbations, due to the changing binocular disparities, in the positions of dots on the monitor. The potential disruptive effect of these perturbations was reduced by the fact that they were distributed equally across both the left and the right views of the stereo pair. In other words, we computed the correct velocities for a cyclopean view of the object and then slightly altered the position of each dot in both stereo views to create a set of binocular disparities consistent with the stereo-defined surface. The consequence of these perturbations is that a small amount of nonrigidity is introduced into the motion. To determine if this disrupted the perception of structure from motion, we visually compared monocular views of stereo-motion conflict displays with stereo-motion consistent displays. The result of our informal observations was no increase in perceived nonrigidity when the perturbations from the conflicting binocular disparities were introduced.

These stereo-motion consistent displays actually played a more fundamental role in our experiments: The observers used them as a matching stimulus to judge the 3-D structure of the conflicting stimuli. The observers could toggle back and forth between the conflicting and the consistent stereo-motion displays by pushing a mouse button; their task in all three experiments was simply to adjust the consistent display until it matched the conflicting display. Unlike the conflict stimuli, these displays had the same constant angular velocity on every trial $\left(1.0^{\circ}\right.$ per frame), but their phase was randomly determined so that the observers could not directly compare 2-D velocities for comparable points in the rotation sequence. In Experiment 1, the observers adjusted the shape index of the matching stimulus. In Experiment 2, they adjusted its curvedness. In Experiment 3, the observers were required to adjust the principal curvatures $\left(k_{1}\right.$ and $\left.k_{2}\right)$ directly.

\section{EXPERIMENT 1 Judged Shape Index From Binocular Stereopsis and Kinetic Depth}

In Experiment 1, we measured the perception of shape index for stereo-motion conflict displays. Earlier studies have examined the perception of shape index from either binocular stereopsis (de Vries et al., 1993) or motion (van Damme et al., 1994), but the experiment reported here is the first to examine the simultaneous contribution of both sources to perceived shape index. Thus, we sought to measure the relative contribution of motion and binocular stereopsis to the perception of a surface's shape index and then compare that with the stereo and motion contribution to judged curvedness that would be measured in Experiment 2.

\section{Method}

Our observers judged the shape index of 200 quadric surface patches as described by Equation 1. All these surfaces had the same curvedness $\left(0.75 \mathrm{~cm}^{-1}\right)$, but, on each trial a different shape index was randomly chosen (from the interval $0.0-1.0$ ) for the binoculardisparity-defined surface and the motion-defined surface. Examples of two surfaces with the same curvedness but different shape index values can be seen in Figure 3.

At the beginning of each trial, the stereo-motion conflict display appeared. The observer would then toggle back and forth between 
this display and the stereo-motion consistent adjustment display. By moving the mouse, the observers could change the simulated shape index of the matching display, and they could toggle between the conflicting and consistent stereo-motion displays until they were satisfied with their match. They then pressed another button on the mouse, thus recording their response and beginning the next trial.

\section{Results and Discussion}

In Experiment 1, the observers were asked to judge the shape index of stereo-motion conflict displays by adjusting a stereo-motion consistent display until a match was achieved. Thus, for every trial, we can express our observers' judged shape index as a function of both the stereo-defined and the motion-defined shape index values based on the following simple model:

$$
S I_{\text {judged }}=w_{\mathrm{s}} * S I_{\mathrm{s}}+w_{\mathrm{m}} * S I_{\mathrm{m}},
$$

where $S I_{\text {judged }}$ is the subjects' adjusted shape index value, $S I_{\mathrm{s}}$ and $S I_{\mathrm{m}}$ are the simulated shape index specified by binocular stereopsis and motion, and $w_{\mathrm{s}}$ and $w_{\mathrm{m}}$ are the stereo and motion weights. A multiple linear regression was performed on these data to determine to what extent this model could account for the integration of stereo and motion information. The results of this analysis for each of our observers can be seen in Table 1. We can see from the large $R^{2}$ values that the linear model provides an excellent fit for the observers' shape index judgments.

The finding that a linear relation accurately describes the integration of multiple 3-D sources is consistent with a number of previous studies (e.g., Bruno \& Cutting, 1988; Bülthoff \& Mallott, 1988). For the specific case of perceived 3-D structure from motion and binocular stereopsis, a number of researchers have also reported linearity in integration for at least some stimulus conditions (Dosher, Sperling, \& Wurst, 1986; Johnston, Cumming, \& Landy,
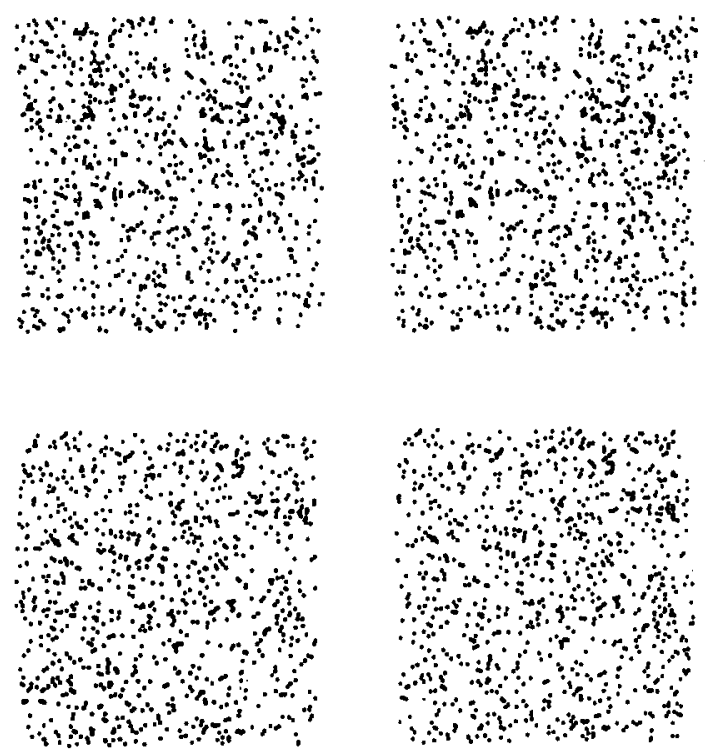

Figure 3. Sample stereograms depicting two quadric surfaces with the same curvedness but different shape index values: top (0.0) and bottom (1.0). The stereogram is designed for cross-fusion.
Table 1

Results of the Multiple Regression Analysis for the Shape Index Judgments From Experiment 1

\begin{tabular}{lccc}
\hline Subject & Multiple $R^{2}$ & $t$ (Motion Coefficient) & $t$ (Stereo Coefficient) \\
\hline J.F.N. & $.95(.14)$ & $24.16^{*}$ & $19.19^{*}$ \\
V.J.P. & $.95(.13)$ & $36.41^{*}$ & $8.55^{*}$ \\
H.F.N. & $.98(.07)$ & $14.92^{*}$ & $64.65^{*}$ \\
J.S.T. & $.98(.08)$ & $62.39^{*}$ & $14.50^{*}$ \\
\hline
\end{tabular}

Note-Standard deviations of the multiple $R^{2}$ appear in parentheses. ${ }^{*} p<.001$.

1994; Rogers \& Collett, 1989; Tittle \& Braunstein, 1991). However, significant nonlinearities in stereo-motion integration have been observed for both transparent stereograms (Tittle \& Braunstein, 1993) and near-threshold stimuli (Bradshaw \& Rogers, 1992; Tittle, Perotti, \& Norman, 1997).

The data in Table 1 also clearly show that both motion and binocular disparity make a significant contribution to the perceived shape index in the stereo-motion conflict displays. The relative contribution of the two sources can be seen from the standardized regression coefficients (displayed as the striped bars in Figure 5). From this figure, it can be seen that, for Observers J.S.T., V.J.P., and J.F.N., motion contributed more to judged shape index than did stereo; however, for Observer H.F.N., the reverse was true. Because previous stereo-motion studies looked only at scale-dependent aspects of 3-D structure, it is difficult to put this specific result in context. However, our primary interest here is in comparing the relative contributions of motion and stereo to the scale-independent (i.e., shape index) and scale-dependent (i.e., curvedness) aspects of 3-D structure. So, in Experiment 2, we measured judged curvedness for stereo-motion conflict displays.

\section{EXPERIMENT 2 Judged Curvedness From Binocular Stereopsis and Kinetic Depth}

\section{Method}

As in Experiment 1, the observers judged the 3-D structure of 200 quadric surfaces. However, in Experiment 2, the shape index simulated by motion and stereo was identical for each trial. This shape index was always one of five values $(0.0,0.25,0.5,0.75$, and $1.0)$, and each of these specific values was presented for one fifth of the total trials in the experiment. Although the two sources simulated the same shape index on each trial, a different curvedness was randomly chosen (from the interval $0.25 \mathrm{~cm}^{-1}-1.25 \mathrm{~cm}^{-1}$ ) for the binocular-disparity-defined surface and the motion-defined surface. Sample stereograms of two surfaces with the same shape index but different curvedness values can be seen in Figure 4. The observers' task was similar to that described in Experiment 1, except now they had to adjust the curvedness of the stereo-motion consistent stimulus until it matched the conflicting display.

\section{Results and Discussion}

In Experiment 2, the observers judged the curvedness of stereo-motion conflict displays. These judged values were then regressed against the simulated stereo and motion curvedness on the basis of the following simple linear model: 


$$
C_{\text {judged }}=w_{\mathrm{s}} * C_{\mathrm{s}}+w_{\mathrm{m}} * C_{\mathrm{m}},
$$

where $C_{\text {judged }}$ is the subjects' adjusted curvedness value, $C_{\mathrm{s}}$ and $C_{\mathrm{m}}$ are the simulated shape index specified by binocular stereopsis and motion, and $w_{\mathrm{s}}$ and $w_{\mathrm{m}}$ are the stereo and motion weights. The data in Table 2 demonstrate that, as in Experiment 1, the linear model provides a very good description of the integration of stereo and motion. As in Experiment 1, we found that both stereo and motion significantly contributed to the judged 3-D structure in the conflict stimulus. However, looking at the standardized regression coefficients (the solid bars in Figure 5), we can see that, for all 4 observers, stereo contributed significantly more to judged curvedness than did motion. This particular result is consistent with most previous studies of stereo-motion integration (Dosher et al., 1986; Rogers \& Collett, 1989; Tittle \& Braunstein, 1991).

Our primary interest in conducting these experiments was to determine whether the relative stereo and motion contributions vary depending on whether observers judge shape index or curvedness. To that end, we have plotted the standardized regression coefficients for motion on the left side of each graph in Figure 5 and the stereo coefficients on the right side. For 3 of our observers the pattern is clear: Motion contributed significantly more to judged shape index than it did to judged curvedness, and stereo contributed significantly more to judged curvedness than it did to judged shape index. Observer H.F.N. also showed this basic pattern, but the differences were small and did not exceed the $95 \%$ confidence interval for the weight estimates. It seems that Observer H.F.N. may also show this effect but a strong overall stereo dominance is masking the result. If the differences between conflicting inter-
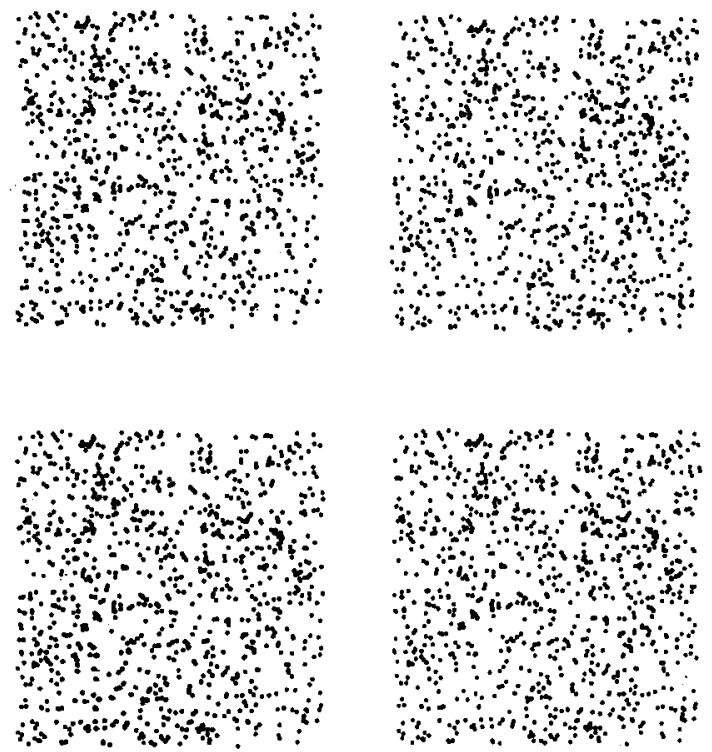

Figure 4. Sample stereograms depicting two quadric surfaces with the same shape index $(0.0)$ but different curvedness values. The stereogram is designed for cross-fusion.
Table 2

Results of the Multiple Regression Analysis for the Curvedness Judgments From Experiment 2

\begin{tabular}{lccc}
\hline Subject & Multiple $R^{2}$ & $t$ (Motion Coefficient) & $t$ (Stereo Coefficient) \\
\hline J.F.N. & $.98(.08)$ & $21.04^{*}$ & $48.08^{*}$ \\
V.J.P. & $.96(.12)$ & $16.41^{*}$ & $26.75^{*}$ \\
H.F.N. & $.98(.08)$ & $12.20^{*}$ & $59.99^{*}$ \\
J.S.T. & $.97(.11)$ & $17.41^{*}$ & $27.75^{*}$ \\
\hline
\end{tabular}

Note-Standard deviations of the multiple $R^{2}$ appear in parentheses. ${ }^{*} p<.001$.

pretations are too large to be meaningfully resolved, the system may be forced into quasi-veto mode in which responses are primarily based on only one source of information. In Experiment 3, we attempted to alleviate this problem by constraining the maximum difference between the motion and stereo surfaces on any given trial.

Although we believe that these regression coefficient shifts represent a fundamental difference in the degree to which motion and stereo specify scale-dependent versus scale-independent aspects of 3-D structure, we must be careful to consider simpler interpretations. For example, in each of the first two experiments, the stimulus set and the subjects' responses varied on only a single dimension: shape index for Experiment 1 and curvedness for Experiment 2. Thus, it is possible that the differences that we see in Figure 5 may only occur because we forced the observers to use one dimension to make a multidimensional match.

By artificially forcing the observers to fit their perceptions into either the shape index or curvedness scales, it may have been the case that the surface actually seen by an observer was not part of the 1-D family of surfaces available in the adjustment stimulus. In an attempt to rule out such possibilities, we conducted Experiment 3, in which the stereo and motion surfaces could simultaneously differ in terms of both shape index and curvedness. We also developed a new response stimulus that allowed the observers to independently adjust the principal curvatures so that their responses would be less constrained.

\section{EXPERIMENT 3 Judged Principal Curvature From Binocular Stereopsis and Kinetic Depth}

In Experiments 1 and 2, we have found evidence that motion contributes more to shape index and stereo contributes more to curvedness. However, because the observers performed very different tasks in Experiments 1 and 2, it is impossible to know whether or not this finding is simply a result of differing demand characteristics for our two tasks. Thus, in Experiment 3, the stereo and motion surfaces simultaneously differed on both shape index and curvedness, and the observers directly adjusted the two principal curvatures in the matching stimulus. This allows us to compute the judged shape index and curvedness from the observers' adjusted $k_{1}$ and $k_{2}$ values and thus estimate the relative contributions of motion and stereo independent of task differences. In ad- 


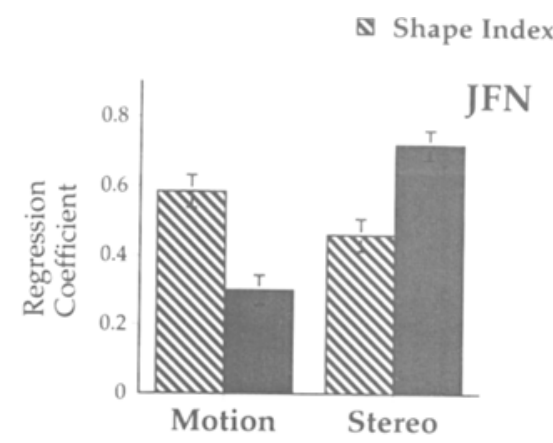

- Curvedness
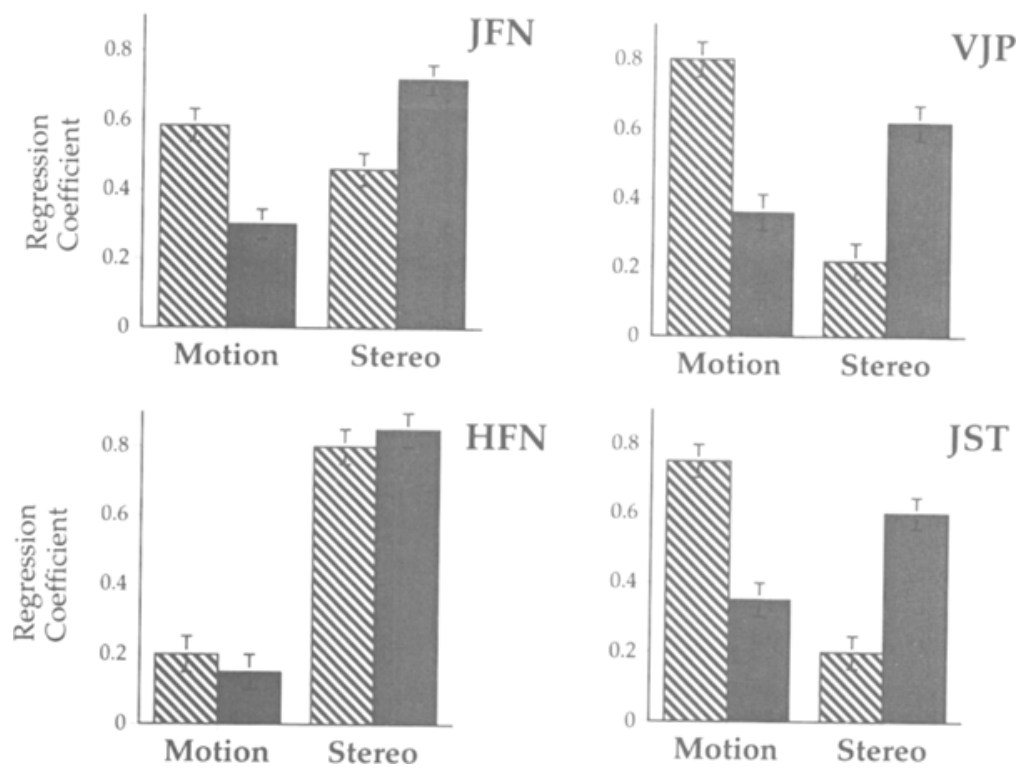

Figure 5. Bar graphs showing the standardized regression coefficients of motion and stereo in Experiment 1 (judged shape index) and Experiment 2 (judged curvedness). Error bars represent the $95 \%$ confidence intervals of the regression coefficient.

dition, to reduce any tendency for the observers to rely too heavily on one source of information (as in the case of H.F.N.), we forced the stereo and motion surfaces to have the same sign of Gaussian curvature on every trial.

\section{Method}

As in Experiments 1 and 2, the observers judged the 3-D structure of 200 quadric surfaces. However, in Experiment 3, the stereoand motion-defined surfaces differed in terms of both shape index and curvedness. This was accomplished by randomly choosing a motion $\left(k_{1}, k_{2}\right)$ and a stereo $\left(k_{1}, k_{2}\right)$ for each trial such that the curvedness fell within the range $0.25 \mathrm{~cm}^{-1}-1.25 \mathrm{~cm}^{-1}$ and the shape index was within the range $0.0-1.0$. Furthermore, the principal curvatures were constrained so that the motion and stereo shape index values were both either between 0.0 and 0.49 or between 0.51 and 1.0. The purpose of this constraint was to force the sign of Gaussian curvature to be the same for both the stereo- and motion-defined surfaces on every trial. The task in this experiment was a 2-D match in which a horizontal movement of the mouse adjusted one principal curvature of the response stimulus and a vertical movement adjusted the other. Using these adjusted principal curvature values, we could compute the judged shape index and curvedness from the identical task.

\section{Results and Discussion}

For each trial in Experiment 3, our observers adjusted $k_{1}$ and $k_{2}$ of the stereo-motion consistent stimulus to match the perceived 3-D structure of the conflict stimulus. Using Equations 5 and 6, we then computed the judged shape index and curvedness for each trial. Two separate regression analyses were performed on these data: the first based on the computed shape index and the second based on the computed curvedness. The results from the shape index analysis can be seen in Table 3 , and those from the curvedness analysis can be seen in Table 4. As in Experiments 1 and 2, we again found that the linear model described the data quite well and that both stereo and motion significantly contributed to judged shape index and curvedness.

However, more importantly, we can see from Figure 6 that the basic pattern of the standardized regression coefficients was comparable to that observed in Experiments 1 and 2. Specifically, for all observers, motion contributed significantly more to judged shape index than it did to judged curvedness, and stereo contributed significantly more to judged curvedness than it did to judged shape index. This result is particularly important since it cannot be explained by differences in the response scale or the task, because the shape index and curvedness values were computed from the same adjusted $k_{1}$ and $k_{2}$ on each trial. Thus, we can conclude that the differences between the contributions of stereo and motion to shape index and curvedness are indicative of the information about 3-D structure derived from these two sources.

Given that this result did not depend on forcing the observers to use a 1-D response set, can we then conclude that it is indicative of a fundamental difference between the specification of scale-dependent and scale-independent aspects of 3-D structure in the visual system? One way to address this question is to analyze the same data presented in Figure 6 but with a parameterization of the surface that does not separate out the scale-dependent and scale- 
Table 3

Multiple Regression Analysis for the Shape Index Values Computed From the Simulated Motion and Stereo Principal Curvatures and From the Observers' Adjusted Principal Curvatures in Experiment 3

\begin{tabular}{lccc}
\hline Subject & Multiple $R^{2}$ & $t$ (Motion Coefficient) & $t$ (Stereo Coefficient) \\
\hline J.F.N. & $.97(.11)$ & $13.89^{*}$ & $3.90^{*}$ \\
V.J.P. & $.96(.12)$ & $10.42^{*}$ & $6.34^{*}$ \\
H.F.N. & $.97(.11)$ & $6.88^{*}$ & $12.08^{*}$ \\
J.S.T. & $.98(.10)$ & $12.50^{*}$ & $7.89^{*}$ \\
J.T.T. & $.97(.10)$ & $8.78^{*}$ & $9.38^{*}$
\end{tabular}

Note-Standard deviations of the multiple $R^{2}$ appear in parentheses. ${ }^{*} p<.001$.

Table 4

Multiple Regression Analysis for the Curvedness Values Computed From the Simulated Motion and Stereo Principal Curvatures and From the Observers' Adjusted Principal Curvatures in Experiment 3

Subject Multiple $R^{2} \quad t$ (Motion Coefficient) $t$ (Stereo Coefficient)

J.F.N. $.96(.09) \quad 6.87^{*} \quad 8.79^{*}$

$\begin{array}{llll}\text { V.J.P. } & .96(.12) & 10.42^{*} & 6.34^{*}\end{array}$

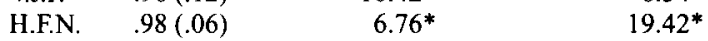

$\begin{array}{llll}\text { J.S.T. } & .98(.06) & 10.31^{*} & 14.70^{*}\end{array}$

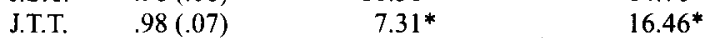

Note-Standard deviations of the multiple $R^{2}$ appear in parentheses. ${ }^{*} p<.001$.

independent components of 3-D structure. Of course, this is easily done, because there exist many $2-D$ parameterizations of a smooth surface. However, a well-known representation of surface structure that can be directly computed from $k_{1}$ and $k_{2}$ is provided by the Gaussian curvature $(K)$ and the mean curvature $(H)$ :

$$
K=k_{1} k_{2}
$$

and

$$
H=\frac{k_{1}+k_{2}}{2} \text {. }
$$

The Gaussian curvature and the mean curvature provide a complete specification of the local second-order structure of a smooth surface; however, unlike shape index and curvedness, they confound the scale-dependent and scale-independent aspects of surface structure. Figure 7 shows the standardized regression coefficients resulting from a multiple regression analysis of the $k_{1}$ and $k_{2}$ responses transformed by Equations 7 and 8 . Although the specific $R^{2}$ values are not presented here, we again found that the linear model accounted for most (at least $96 \%$ ) of the variance in our observers' responses. However, the important point is that the two motion regression coefficients and the two stereo regression coefficients are not significantly different for any of our observers. In other words, when we analyze the $k_{1}$ and $k_{2}$ data using a surface parameterization that confounds scale-dependent and scale-independent aspects of 3-D structure, the difference between the relative contributions of motion and stereo observed in Experiments 1 and 2 disappears. This strongly suggests to us that the differences in the relative contribution of motion and stereo reported here occurred because these two sources do not equally specify the scaledependent and scale-independent aspects of surface structure.

\section{GENERAL DISCUSSION}

The three experiments presented here were designed to examine how the integration of binocular stereopsis and perceived kinetic depth depend on the surface properties being measured. We specifically measured observers' judgments for shape index and curvedness because previous empirical results have suggested that these properties may not be equally specified by binocular disparities and relative velocities. In Experiment 1, observers matched the perceived shape index of a stereo-motion conflict stimulus with that of a stimulus in which the stereo and motion information were consistent. In Experiment 2, observers matched the apparent curvedness of the conflicting and consistent stereo-motion stimuli. Finally, in Experiment 3, observers matched the principal curvatures (effectively adjusting shape index and curvedness simultaneously) of the conflicting and consistent stereo-motion stimuli. The results of these experiments indicate that the integration of binocular stereopsis and perceived kinetic depth significantly varies as a function of the specific surface properties being measured.

For motion, we found a greater contribution to judged shape index than to judged curvedness. On the other hand, binocular stereopsis contributed more to curvedness than to shape index. This pattern of results was observed in Experiments 1 and 2, in which observers adjusted either shape index or curvedness, and in Experiment 3, in which observers adjusted the principal curvatures directly. Thus, these differences cannot be explained by any demand characteristics introduced because of the specific response that observers were asked to perform.

If the difference between the relative contributions of binocular stereopsis and perceived kinetic depth are not due to task differences, what might be causing the results reported here? At least a partial answer can be provided by a recent theoretical result from Dijkstra, Snoeren, and Gielen (1994). For perceived structure from motion, Dijkstra et al. found that one can recover the shape index of a local region from the second-order differential invariants of the velocity field. However, to compute the curvedness, one must also recover a scaling parameter: the 3-D velocity of the object or the observer. Using only the image velocity field, this cannot be accomplished, and thus such an analysis predicts that motion should be a more salient cue for shape index than for curvedness. Although it is important to note that, using the acceleration field, one could compute local curvedness, recent empirical studies of structure from motion (e.g., Liter, Braunstein, \& Hoffman, 1993; Todd \& Norman, 1991) 

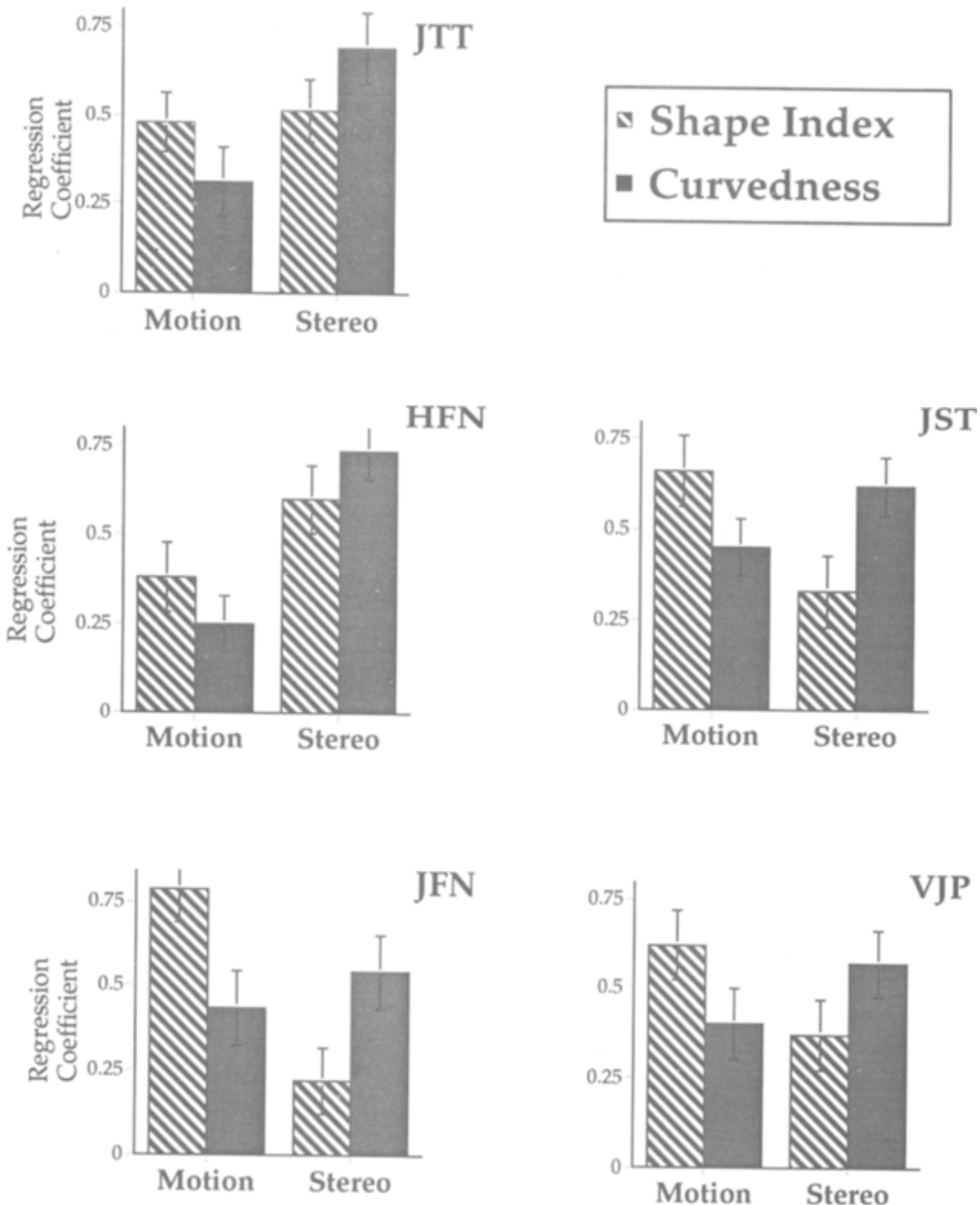

Figure 6. Bar graphs showing the standardized regression coefficients of motion and stereo for judged shape index (striped bars) and judged curvedness (solid bars). Shape index and curvedness were computed from the observers' adjusted $k_{1}$ and $k_{2}$. Error bars represent the $95 \%$ confidence intervals of the regression coefficient.

indicate that observers' 3-D judgments can be accounted for by the information contained in two frame motion sequences (or velocity fields). The results of these studies suggest that observers use some heuristic to determine depth scale rather than apply an analysis based on the acceleration field.

Perceived curvedness from binocular stereopsis also requires some scaling information, but, unlike for the motion, there are some potential sources for scaling the binocular disparity field. Specifically, the magnitude of curvedness can be recovered given an accurate estimate of the fixation distance or interocular separation. Despite the existence of such information, numerous psychophysical studies have established that perceived depth from binocular stereopsis is scaled by fixation distance, but this scaling is not veridical (e.g., Johnston, 1991). Johnston et al. (1994) and Richards (1985) have argued that motion could provide sufficient information to scale binocular disparities accurately. However, previous studies of perceived 3-D structure from the combination of motion and disparity do not support the contention that motion information allows for veridical stereo scaling (Norman, Todd, Perotti, \& Tittle, 1996; Norman, Todd, \& Phillips, 1995; Rogers \& Collett, 1989; Tittle \& Braunstein, 1993; Tittle et al., 1995).

Previous studies of the integration of motion and binocular stereopsis have considered only scale-dependent aspects of 3-D shape, but, to the extent that their stimulus conditions overlap with our curvedness manipulation, the results are consistent with those reported here. Rogers and Collett (1989) and Tittle and Braunstein (1991) measured judged 3-D shape for motion-stereo conflict stimuli that differed in the magnitude of simulated depth along the line of sight. In both of these studies, it was observed that when motion and binocular disparity were placed in conflict, the resulting perceived depth was de- 


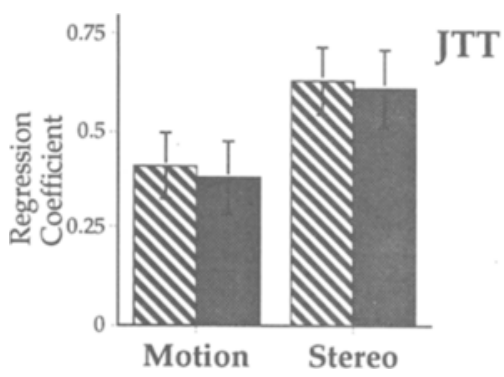

s Gaussian Curvature - Mean Curvature
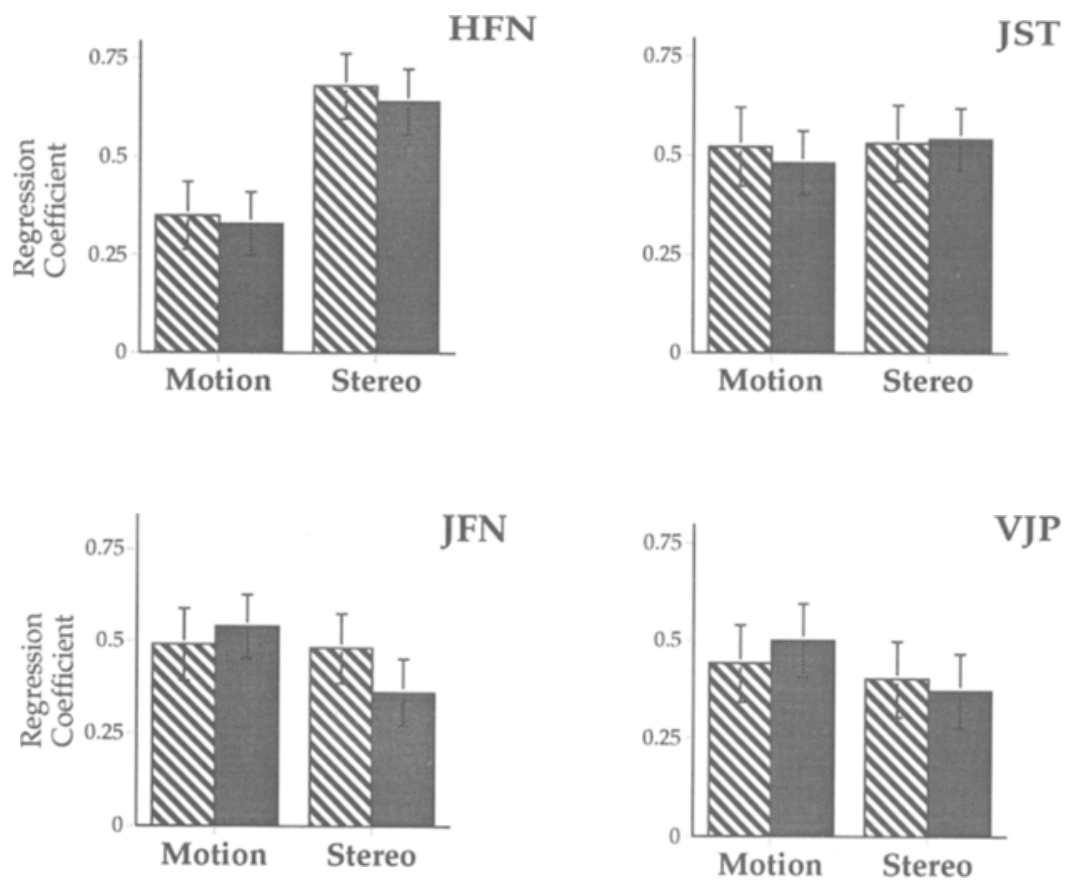

Figure 7. Bar graphs showing the standardized regression coefficients of motion and stereo for judged Gaussian curvature (striped bars) and judged mean curvature (solid bars). Gaussian curvature and mean curvature were computed from the observers' adjusted $\boldsymbol{k}_{1}$ and $k_{2}$. Error bars represent the $95 \%$ confidence intervals of the regression coefficient.

termined primarily by the binocular disparity information. This result coincides with our finding that binocular stereopsis contributes more to curvedness judgments than does perceived kinetic depth.

Johnston et al. (1994) examined the judged depth of cylindrical surfaces in which the depth-to-height ratio specified by motion and stereo were in conflict. These experiments are the equivalent of our curvedness manipulations for cylindrical surfaces (shape index $=0.5$ ), as shown in Figure 2. For relatively short viewing distances $(50 \mathrm{~cm})$ and for two frame motion stimuli, these researchers also found that stereo contributed more heavily to judged depth than did motion. However, unlike the results reported here or those of Rogers and Collett (1989) and Tittle and Braunstein (1991), Johnston et al. found that, at far viewing distances $(200 \mathrm{~cm})$, the average weight attributed to motion was over twice that for binocular disparity. Because they used a scale-dependent measure of 3-D structure, it is difficult to know whether this result represents nonveridical scaling of binocular disparities at far viewing distances or an actual change in the relative importance of these two sources. The shape index and curvedness distinction described here may provide an interesting way to untangle these two possibilities, because the scale-independent component of 3-D structure (shape index) should not vary as a function of viewing distance even if depth from disparity is scaled inappropriately.

The results we have described provide specific information about how binocular stereopsis and kinetic depth contribute to two different aspects of 3-D structure. However, we believe that these data also relate to a much more general and fundamental issue regarding the plausibility of certain classes of shape integration models. Specifically, they are directly relevant to the issue of what exactly is being integrated from the various optical sources of information. Current theories of 3-D structure integration are primarily based on the assumption that there is a single common representation at which all the out- 
puts from the various "structure-from- $X$ " processes are combined (e.g., Bülthoff \& Yuille, 1991; Landy, Maloney, Johnston, \& Young, 1995).

Typically, this common representation is assumed to be a depth map, but such a metric representation cannot easily account for the prominence of ordinal cues, such as occlusion in the perception of 3-D structure (Braunstein, Andersen, Rouse, \& Tittle, 1986). One solution to this problem has been proposed by Cutting and Vishton (1995), who argue that one can first compute an ordinal representation of depth from all available sources and then derive a metric from the set of pointwise comparisons. However, it is our contention that, regardless of the nature of the representation, any model that assumes that the output of all structure-from- $X$ processes first contribute to a single representation may not be compatible with human perception in multiple-cue conditions.

The diagram at the top of Figure 8 is intended as a simplified schematic of a representational framework in which a structure-from-motion and a structure-fromstereopsis process each compute a local depth map after some initial interactions. If we assume comparable scaling of perceived kinetic depth and binocular stereopsis and a final linear integration, after some potential early nonlinearity (as proposed by Landy et al., 1995), then the relative contributions of motion and stereopsis are determined by the 2-D weight vector $\left(\mathbf{W} \mathbf{M}_{\mathbf{d}}, \mathbf{W S}_{\mathbf{d}}\right)$. So far, all of this is perfectly consistent with the results reported here, because after integrating our two sources to get a depth map, we can simply compute local curvedness and shape index as shown in Equations 3-6. However, the difficulty arises when we consider what would be the relative contribution of motion and binocular stereopsis to these computed shape index and curvedness values. If these measures are computed from the same depth map at the top of Figure 8, then it follows that they should each have the identical weight vector $\left(\mathbf{W} \mathbf{M}_{d}, \mathbf{W S} \mathbf{S}_{\mathbf{d}}\right)$, which describes the relative contribution of motion and stereopsis to depth. This prediction is not consistent with our findings that motion contributes more to shape index and stereo contributes more to curvedness.

Instead of models that assume a single initial representation, we would argue that our data are more consistent with an approach represented by the diagram at the bottom of Figure 8. Again, we have two structure-from$X$ processes, but, in this case, they each have multiple outputs: depth, curvedness, and shape index. If we assume that the depth map is not a special first step in the computational process but rather just another possible local representation, the difficulty about predicting the same weight vector for the curvedness and shape index values does not occur. As can be seen at the bottom of Figure 8, we instead expect a unique weight vector for each of the three local 3-D properties being computed: depth $\left(\mathbf{W M}_{\mathbf{d}}\right.$, $\left.\mathbf{W S}_{\mathbf{d}}\right)$, curvedness $\left(\mathbf{W} \mathbf{M}_{\mathfrak{c}}, \mathbf{W S}\right)$, and shape index $\left(\mathbf{W M} \mathbf{M}_{\mathbf{S I}}\right.$, $\mathbf{W S}_{\mathrm{SI}}$ ). We would thus argue that our data suggest that theories that do not assume a single initial representation
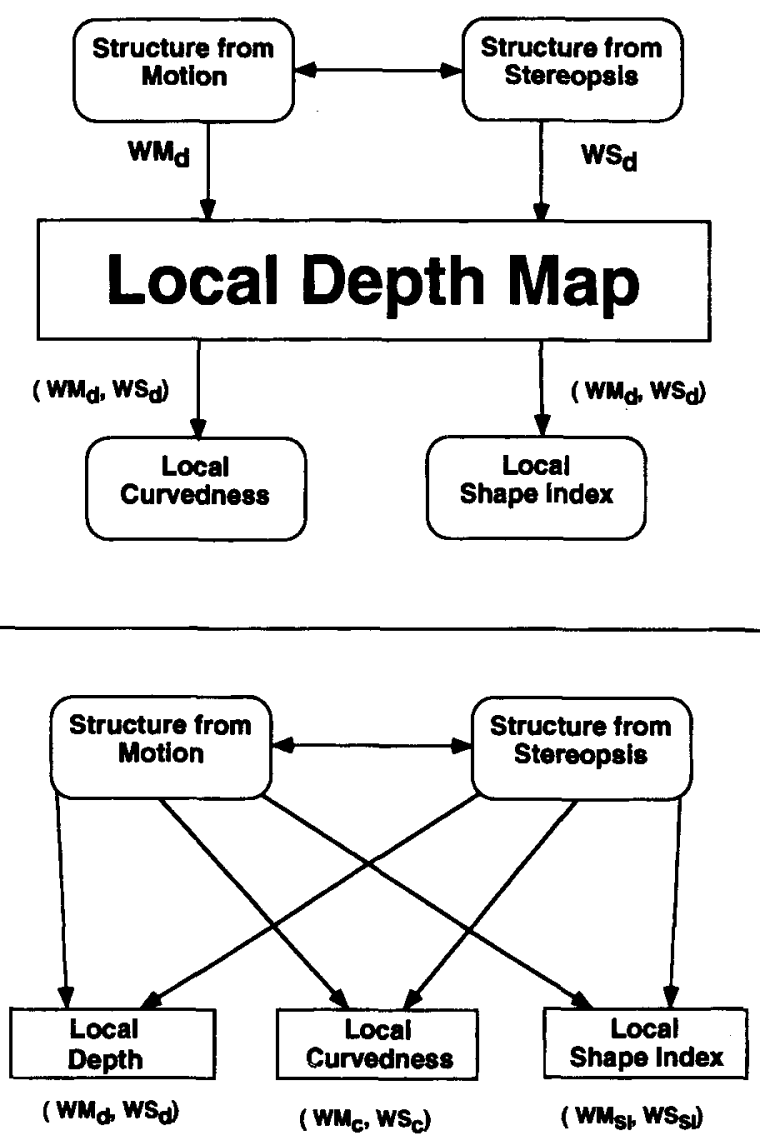

Figure 8. Diagram showing schematic views of (top) an integration scheme in which stereo and motion combine their outputs at a common depth map stage and (bottom) an integration scheme in which stereo and motion combine their outputs into local depth, shape index, and curvedness representations with no intervening common stage.

for all sources of 3-D structure come closest to describing how human observers use multiple visual cues.

The preceding discussion began with the assumption of comparable scaling of perceived kinetic depth and binocular stereopsis. However, it is important to consider how this interpretation of the weight values would change if we do not assume approximately equal scaling. Considering the quadric surface specified in Equation 1, it can be seen that the values $k_{1}$ and $k_{2}$ could be scaled differentially for motion $\left(s_{\mathrm{m}}\right)$ and binocular stereopsis $\left(s_{\mathrm{s}}\right)$. If it were the case that $s_{\mathrm{m}}=s_{\mathrm{s}}$, we could readily interpret the weights shown in Figures 5 and 6 as the relative contributions of motion and binocular stereopsis to judged curvedness and shape index. However, there are a number of studies suggesting that the scaling of motion and stereo is not equivalent (e.g., Rogers \& Graham, 1983; Tittle et al., 1995); thus, we will describe the possible effects of this on the relative weights measured for shape index and curvedness.

The most important consequence of differences between motion and stereo scaling occurs because one of 
our measurements of 3-D structure is scale independent (shape index) and the other is scale dependent (curvedness). It can be shown that scale plays no role in specifying the local shape index because taking the ratio of the principal curvatures $k_{1}$ and $k_{2}$, as shown in Equation 7, cancels out the scale applied to each principal curvature. Thus, it turns out that the weights for the curvedness judgments are scale dependent, but the weights obtained for shape index judgments are not. To see the consequences of this, consider two new variables that equal the relative contribution of binocular stereopsis and perceived kinetic depth to shape index $\left(R C_{S I}=W S_{S I} / W M_{S I}\right)$ and curvedness $\left(R C_{C}=W S_{C} / W M_{C}\right)$. Now, because $R C_{S I}$ is scale dependent and $R C_{C}$ is not, we would expect the following simple relation to hold if the relative contribution of disparity and motion were actually the same for shape index and curvedness: $R C_{S I}=R C_{C} *\left(s_{\mathrm{s}} / s_{\mathrm{m}}\right)$.

For the data in Experiment 3, the average value of the scaling of stereo relative to motion $\left(s_{\mathrm{s}} / s_{\mathrm{m}}\right)$ would have to be approximately 2.3 in order for the contribution of stereo and motion $\left(R C_{S I}\right.$ and $\left.R C_{C}\right)$ to be equal for judgments of shape index and curvedness. The question that must now be addressed is whether this value of relative scaling is plausible for our shape matching task. Some recent data collected by Perotti et al. (in press) provide us with an opportunity to answer this question. These authors had observers match the curvedness of a motion-defined surface to the curvedness of a stereo-defined surface under conditions almost identical to those reported in this paper. The slope of the function relating adjusted motion curvedness to adjusted stereo curvedness provides an estimate of $s_{\mathrm{s}} / s_{\mathrm{m}}$, and, for their 4 observers, the average slope equaled approximately 1.5 . Although this estimate of the relative scaling is lower than that required to predict equal contributions of motion and stereo in our experiment, the values are close enough that this possibility cannot be ruled out altogether.

Even though an interpretation of our results based on differences in the relative scaling of motion and stereo is possible, recent studies support our claim that higher order aspects of surface structure need not be computed from a depth map. Both Johnston and Passmore (1994) and Norman and Todd (in press) have provided evidence indicating that higher level perceptual representations (e.g., local slant or curvature) are not computed from an initial representation based on a depth map. Furthermore, Koenderink and van Doorn (1995) have shown that observers are more accurate at judging the orientation of widely separated surface regions than they are at judging the depth. The results of these studies coupled with the findings of our experiments provide a strong case against theories of cue integration based on an initial depth map.

Finally, it is worth noting that the argument outlined above does not depend on whether or not shape index and curvedness have some special perceptual status. The results of Experiment 3 seem to indicate that there is some perceptual reality to a surface parameterization based on scale-dependent and scale-independent components. Nevertheless, our conclusion concerning the inadequacy of integration models that assume a single initial representation stage applies regardless of whether that initial representation is local orientation, curvature, or any other single surface property. Instead, we argue that a model capable of explaining the complexity of perceived 3-D structure from multiple sources must incorporate the many 3-D structural attributes that make up our perception of objects and surfaces.

\section{REFERENCES}

Bradshaw, M. F., \& Rogers, B. J. (1992). Subthreshold interactions between binocular disparity and motion parallax. Investigative Ophthalmology \& Visual Science, 33, 1332.

Braunstein, M. L., Andersen, G. J., Rouse, M. W., \& Tittle, J. S. (1986). Recovering viewer-centered depth from disparity, occlusion, and velocity gradients. Perception \& Psychophysics, 40, 216-224.

BRUNo, N., \& CUTtiNG, J. E. (1988). Minimodularity and the perception of layout. Journal of Experimental Psychology: General, 117, 161-170.

BÜLTHOFF, H. H., \& MALLOTT, H. (1988). Integration of depth modules: Stereo and shading. Journal of the Optical Society of America A, 5, 1749-1758.

BülThOFF, H. H., \& Yullle, A. L. (1991). Bayesian models for seeing shapes and depth. Comments Theoretical Biology, 2, 283-314.

Cutting, J. E., \& Vishton, P. M. (1995). Perceiving layout and knowing distances: The integration, relative potency, and contextual use of different information about depth. In W. Epstein \& S. Rogers (Eds.), Handbook of perception and cognition: Vol. 5. Perception of space and motion (pp. 69-117). New York: Academic Press.

DE VRIES, S. C. (1993). Binocular perception of spatial configuration. Doctoral dissertation, Utrecht University, Utrecht, The Netherlands. DE VRIES, S. C., KapPers, A. M. L., \& KoenderinK, J. J. (1993). Shape from stereo: A systematic approach using quadratic surfaces. Perception \& Psychophysics, 53, 71-80.

Dijkstra, T. M. H., Snoeren, P. R., \& Gielen, C. C. A. M. (1994). Extraction of three-dimensional shape from optic flow: A geometric approach. Journal of the Optical Society of America A, 11, 2184-2196.

Dosher, B., Sperling, G., \& Wurst, S. (1986). Tradeoffs between stereopsis and proximity luminance covariance as determinants of perceived 3D structure. Vision Research, 26, 973-990.

Gillam, B., Chambers, D., \& Russo, T. (1988). Postfusional latency in stereoscopic slant perception and the primitives of stereopsis. Journal of Experimental Psychology: Human Perception \& Performance, 14, 163-175.

JoHnSTON, E. B. (1991). Systematic distortions of shape from stereopsis. Vision Research, 50, 1351-1360.

Johnston, E. B., Cumming, B. G., \& LANDY, M. S. (1994). Integration of stereopsis and motion shape cues. Vision Research, 34, 2259-2275.

Johnston, A., \& PASSMORE, P. J. (1994). Independent encoding of surface orientation and surface curvature. Vision Research, 34, 3005-3012.

Koenderink, J. J. (1990). Solid shape. Cambridge, MA: MIT Press.

Koenderink, J. J., \& van Doorn, A. J. (1995). Relief: Pictorial and otherwise. Image \& Vision Computing, 13, 321-333.

KreYsZIG, E. (1959). Differential geometry. Toronto: University of Toronto Press.

Landy, M. S., Maloney, L. T., Johnston, E. B., \& Young, M. (1995). Measurement and modeling of depth cue combination: In defense of weak fusion. Vision Research, 35, 389-412.

Liter, J. C., Braunstein, M. L., \& Hoffman, D. D. (1993). Inferring structure from motion in two-view and multiview displays. Perception, 22, 1441-1465.

Norman, J. F., \& LAPPIN, J. S. (1992). The detection of surface curvature defined by optical motion. Perception \& Psychophysics, 51, 386-396.

Norman, J. F., \& ToDD, J. T. (in press). The discriminability of local surface structure. Perception. 
Norman, J. F., Todd, J. T., Perotti, V. J., \& Tittle, J. S. (1996). The visual perception of 3D length. Journal of Experimental Psychology: Human Perception \& Performance, 22, 173-186.

Norman, J. F., Todd, J. T., \& Phillips, F. (1995). The perception of surface orientation from multiple sources of optical information. Perception \& Psychophysics, 57, 629-636.

Perotti, V. J., Todd, J. T., Lappin, J., \& Phillips, F. (in press). The perception of surface curvature from optical motion. Perception \& Psychophysics.

RICHARDS, W. (1985). Structure from stereo and motion. Journal of the Optical Society of America A, 2, 343-349.

Rogers, B. J., \& ColleTt, T. S. (1989). The appearance of surfaces specified by motion parallax and binocular disparity. Quarterly Journal of Psychology, 41A, 697-717.

Rogers, B. J., \& Graham, M. E. (1983). Anisotropies in the perception of three-dimensional surfaces. Science, 221, 1409-1411.

Tittle, J. S., \& Braunstein, M. L. (1991). Shape perception from binocular disparity and structure from motion. In P. S. Schenker (Ed.), Sensor fusion III: 3-D perception and recognition. Proceedings of the SPIE, 1383, 225-234.

Tittle, J. S., \& Braunstein, M. L. (1993). Recovery of 3-D shape from binocular stereopsis and structure from motion. Perception \& Psychophysics, 54, 157-169.
Tittle, J. S., Perotti, V. J., \& Norman, J. F. (1997). The integration of binocular stereopsis and structure-from-motion in the discrimination of noisy surfaces. Journal of Experimental Psychology: Human Perception \& Performance, 23, 1035-1049.

Tittle, J. S., Todn, J. T., Perotti, V. J., \& Norman, J. F. (1995). The systematic distortion of perceived 3-D structure from motion and binocular stereopsis. Journal of Experimental Psychology: Human Perception \& Performance, 21, 663-678.

TodD, J. T., \& Norman, J. F. (1991). The visual perception of smoothly curved surfaces from minimal apparent motion sequences. Perception \& Psychophysics, 50, 509-523.

TODD, J. T., \& NORMAN, J. F. (1995). The visual discrimination of relative surface orientation. Perception, 24, 855-866.

TODD, J. T., \& REICHEL, F. D. (1989). Ordinal structure in the visual perception and cognition of smoothly curved surfaces. Psychological Review, 96, 643-657.

van Damme, W. J. M., Oosterhoff, F. H., \& van de Grind, W. A (1994). Discrimination of 3-D shape and 3-D curvature in active vision. Perception \& Psychophysics, 55, 340-349.

(Manuscript received February 14, 1996; revision accepted for publication December 9, 1996.) 